2

3

4

5

\title{
CaseSolver: An investigative open source expert system based on EuroForMix
}

\author{
Øyvind Bleka (1*), Lourdes Prieto (2), Peter Gill $(1,3)$ \\ 1 Department of Forensic Sciences, Oslo University Hospital, Oslo, Norway \\ 2 Instituto de Ciencias Forenses, Grupo de Medicina Xenómica, Universidade de Santiago de Compostela, \\ Santiago de Compostela, Spain. \\ 3 Institute of Clinical Medicine, University of Oslo, Oslo, Norway \\ *Corresponding author: E-mail address oyvble@hotmail.com ( $\varnothing$. Bleka), Phone: +47 23013126
}

\section{Abstract}

For very serious crimes, reporting scientists often have to contend with complex cases where literally hundreds of items are submitted by investigators for analysis. In order to efficiently expedite the challenge of comparing reference profiles to evidence profiles, many of which are mixtures, we have developed an investigative open source expert system CaseSolver. We have analysed a real case based on GlobalFiler involving 119 evidence profiles and 3 reference profiles. To provide a demonstration of the power of the system we also added the three references to a fictive large database of 1 million individuals in order to test subsequent recovery of the presumed true contributors. CaseSolver was used on a Fusion $6 \mathrm{C}$ validation study involving 25 two- to four-person mixture profiles based on 14 reference profiles. The sequential use of simple allele comparison, the qualitative model (forensim) and the quantitative model (EuroForMix) makes the analysis very fast and accurate - and finally, the software generates a list of potential match candidates which can be exported as a report. From these two studies we found that the resolution of match candidates from CaseSolver was the same as that reported by a scientist who worked manually through the samples, except that CaseSolver highlighted two manual errors. For the validation study we found low template DNA samples giving negative results, which demonstrate the limitations of the tool; but overall our assessment shows that CaseSolver will benefit all analyses involving mixture interpretation and screening. Importantly, CaseSolver removes the very time-consuming aspect of manual comparison and gives improved quality by preventing manual errors.

\section{Keywords: DNA comparison, Likelihood ratio, Database searching, Expert system}

\section{Introduction}

Commercially available short tandem repeats (STR) amplification kits for forensic casework are becoming more and more comprehensive as the numbers of STR loci are increasing. The first STR multiplexes developed by the Forensic Science Service (FSS) in the United Kingdom in the 1990s consisted of four (Quadruplex) and six (SGM) polymorphic STRs, which provided a matching probability of approximately 1 in 10000 and 1 in 50 million respectively [1,2]. Since then, commercial companies, such as Promega Corporation, Qiagen and ThermoFisher Scientific (former Applied Biosystems and Life Technologies), have developed STR multiplexes for forensic purposes with additional numbers of STR loci, increasing discrimination powers to about 1 in 1 quintillion (1e18) for 15 STRs and 1 in 10 octillion (1e26) for 21 STRs [3]. However, given that such extreme extrapolations of probabilistic models can never be validated, we limit our evaluative statements to 1 in 1 billion. In addition, improvements in biochemistry have resulted in the detection of very low levels of DNA, typically described as low-template analysis $[4,5]$. These tests can be described as both universal and routine. The GlobalFiler ${ }^{\mathrm{TM}}$ PCR Amplification Kit, manufactured by ThermoFisher Scientific and the PowerPlex ${ }^{\circledast}$ Fusion $6 \mathrm{C}$ system by Promega Corporation, consisting of 24 and 27 markers, respectively, includes both the traditional US and European STR loci [6, 7]. These 
amplification kits provide even higher discrimination power, but also enable transatlantic exchange of DNA profiles. The increasing number of additional loci provides more data to manage and causes the interpretation and comparison of DNA profiles to become a time consuming bottle-neck. This can be a challenge in routine casework, especially if multiple stains produce different DNA mixtures of different complexity. Also, when there is little information available about the case circumstances, the list of potential suspects may be vast, as it could comprise individuals held on a national DNA database. The police will expect the scientist to provide investigative leads to identify potential suspects.

A number of statistical software packages are available for the purpose of evaluating the strength of the evidence by calculating the Likelihood Ratio (LR) [8-13]. But before this step is carried out, numerous cross-comparisons of complex crime-stain profiles with reference samples may be needed. The software named eDNA was developed to assist with this task [14]; it automatically provides an overview over all data in casework in a structured manner based on categorization and simple allele comparison. However, the software is not suitable for analysing very large cases or doing database searching, and it does not incorporate any possibility to carry out statistical analysis for mixtures. SmartRank is an open source software for searching DNA databases with complex STR profiles (available at http://Irmixstudio.org). The software implements the forensim model [15-17] that was adapted to enable fast and efficient searching of voluminous databases $[18,19]$. SmartRank presents the top ranking candidate(s) that exceed a predefined LR threshold. Although the software has proven to be useful and efficient in forensic casework, it does not take peak heights into account and it does not provide a structured overview of evidence and reference profiles. Consequently, there is still a need for advanced data management tools that can provide an automated overview of all the evidence profiles (both mixtures and non-mixtures), where the peak height information is taken into consideration, in order to discover potential matches when a large number of references are provided.

In this paper we present CaseSolver (CS), a novel expert system, used to manage comprehensive casework data (open-source and freely available at www.euroformix.com/casesolver). CS provides a graphical user interface in the statistical software $R$ [20] where it can perform rapid comparisons of reference DNA profiles from single source profiles and mixtures. CS follows the method presented by Bleka et al. [21] where a step-wise sequential analysis is performed: First a simple allele comparison is undertaken, followed by analysis with a qualitative model (forensim [15]) and finally a quantitative model (EuroForMix (EFM) [8], which is a similar model as DNAmixtures [13, 22]) is used in order to provide the ultimate list of candidate matches.

To demonstrate how the software can be used as an efficient tool to resolve candidate matches for reporting scientists, we applied CS on a real case (based on GlobalFiler) containing over 100 evidence profiles. In order to test CS more extensively, we generated a large database containing 1 million references and analysed these together with the real case data. Finally, we applied CS on a dataset based on a validation study of the Fusion $6 C$ system conducted in Norway.

\section{Data and experimental design}

\subsection{Circumstances and DNA profiles of a real case}

Following a report from a concerned relative whose sister (REF1) had been missing for several days, the police forcibly entered her residence to find her deceased along with her son (REF2). There had clearly been a violent struggle where the victims had been stabbed multiple times. As the investigators had no indication of a suspect(s) it was decided to carry out comprehensive sampling of the crime scene in order to discover potential suspects from DNA analysis (a map of the crime scene 
is presented in Fig S1 in supplement A.1). Posteriorly, a reference sample from a suspect (REF3) apprehended by the police was collected. A total of 119 crime stains were collected from 46 different items. The majority were blood stains, but contact traces were also recovered from cigarette butts, swabs of mobile telephones and handles of drawers and knife handles and blades that may have been used as weapons by one or more perpetrators and victims (see the list with items and the corresponding DNA profile names in Table S1 in supplement A.1). The DNA profiles were generated using GlobalFiler (ThermoFisher Scientific) and the analytical threshold (AT) was set to $70 \mathrm{rfu}$. More details about the generated profiles are given in supplement A.1. All the DNA profiles (alleles and peak heights) are given at http://www.euroformix.com/datasets. Also provided is a file of allele frequencies used to calculate the LRs [23], based on 284 individuals analysed with GlobalFiler.

\subsection{Searching a large fictive national DNA database}

Often the candidate list of suspects may extend to national DNA databases. A portion of a database may be selected based on geographical location of the offence and known offenders within a given area. In order to test the efficiency of CS, the three profiles of the known persons of interest in the real case, i.e. the two victims and the suspect, were added to 1 mill. randomly generated reference profiles and these were compared to the evidence profiles (based on the crime-stains in the case) using CS. Here the GlobalFiler allele frequencies were used, but in addition we inserted a minimum frequency of 0.0018 for allele 5.3 and allele 28 at the SE33 locus (and afterwards all frequencies were normalized). Importantly, the generation of such a database enables us to count (and estimate) the number of false positives obtained.

\subsection{A validation study based on Fusion $6 C$}

The purpose of the validation study was to investigate how DNA profiles based on the PowerPlex ${ }^{\circledR}$ Fusion $6 C$ (Promega) kit performed with regards to the number of known contributors, mixture proportions, allele drop out, artefacts (stutters/drop in) and mixture interpretation, both manually and with LR calculations using EFM (quantitative model) and LRmix Studio (qualitative model). We applied the samples from the validation study as a case in CS to determine its performance, comprising nine 2-person mixtures, twelve 3-person mixtures and four 4-persons mixtures. These were constructed from 14 good quality single source crime stain samples in different mixture proportions. The 14 single source stains acted as reference samples. The analytical threshold (AT) was set to $100 \mathrm{rfu}$. More details about the profiles are given in supplement A.2. The allele frequencies used to calculate the LR values were based on the Norwegian database found at http://www.familias.no.

\section{Method}

\subsection{Initial analysis}

When CS imports the data (both alleles and peak heights), the software first estimates the minimum number of contributors using the simple rule $K=$ round $U p$ ('maximum allele count for any markers'/2) [24], where roundUp means rounding up to closest integer. Based on this rule, it is possible to assign whether an evidence profile is most likely to be a single source (SS) profile $(K=1)$ or a mixture $(K>1)$. A consequence is that artefacts creating additional alleles like stutters or drop-ins may overestimate the number of contributors. After carrying out 'the number of contributors' classification, CS compares all the imported references against all evidence profiles which were classified as SS profiles (i.e. evidence profiles with $\mathrm{K}=1$ ). If the alleles of the reference (non-empty loci) match with all the alleles of the SS profile, then CS tags the reference name under the "MatchStatus" column of the evidence profile. In order to take into account partial profiles, the user can set a threshold to determine the number of non-empty loci in the SS profile to designate "a 
match" (seven is set as a default). If no reference matched the SS profile, CS creates an "unknown" reference profile with a specific ID. If several of these unknown SS profiles are identical, these will have the same unknown ID. The "MatchStatus" for mixtures is always given as "mixture" (Fig 1).

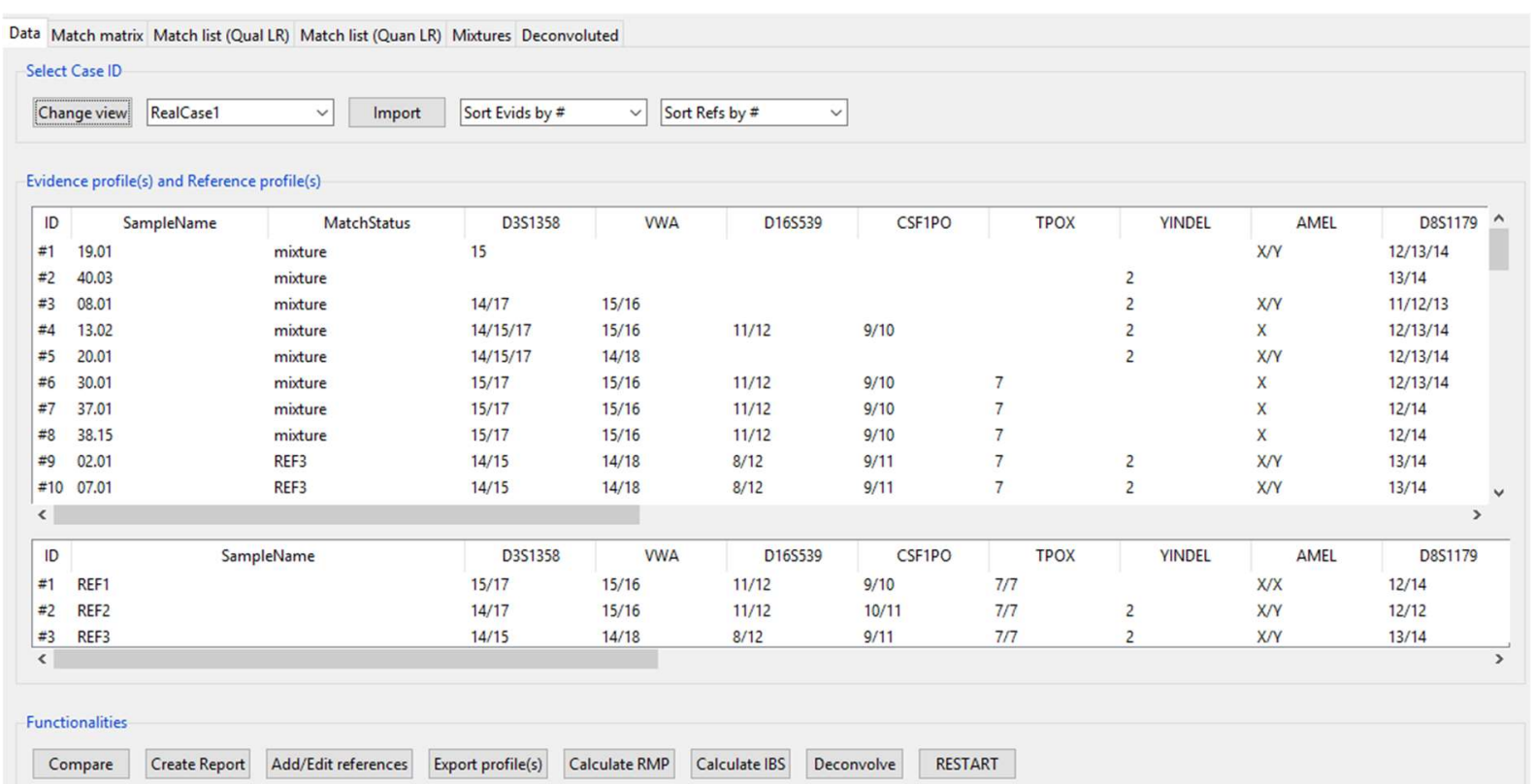

Fig 1: The figure shows the "Data" panel of CS after importing the real case example ("RealCase1"). Here the tables visualize the alleles for each of the profiles, with evidence profiles given in the upper table, and the reference profiles given in the lower table.

\subsection{Mixture comparison}

The total number of comparisons in a case is 'the number of references' multiplied by 'the number of mixtures', and the time taken to perform such a number of comparisons relies heavily upon on the method used. Simple allele comparison, also called "Matching Allele Count" (MAC) [25], is extremely rapid to perform (seconds), whereas inference of the qualitative model would be slower (minutes), and the inference of the quantitative model slower still (hours) [21]. Hence, in order to save computing time, the mixture comparison in CS is based on a three step approach, as described by Bleka et al. [21]. Each step produces a selected list of candidates from the previous step, which are based on user specified thresholds. The first step performs MAC, followed by LR calculations based on the qualitative model (forensim [15]). Finally, LR calculations based on the quantitative model (EFM [8]) were used to filter the list of candidates. Mathematical details for the different models are provided in supplement B.1. We recap how the LR is calculated for both the qualitative and quantitative model:

Consider the two investigative propositions, assuming $K$ number of contributors:

$H_{p}$ : "Person of interest (POI) and $K-1$ unknowns (unrelated) are contributors to evidence profile $E$ "

$H_{d}$ : " $K$ unknowns (unrelated) are contributors to evidence profile $E$ "

The $\mathrm{LR}$ is given by the expression $L R=\frac{P\left(E \mid H_{p}\right)}{P\left(E \mid H_{d}\right)}$. To calculate this, we apply a statistical model $P(E \mid \beta, H)$, with unknown parameters $\beta$ (with $H$ either $H_{p}$ or $H_{d}$ ). By using the maximum likelihood estimate (MLE) approach to infer the unknown parameters $[8,13]$, the LR is given as:

$$
L R=\frac{\max _{\beta} P\left(E \mid \beta, H_{p}\right)}{\max _{\beta} P\left(E \mid \beta, H_{d}\right)}=\frac{L_{p}}{L_{d}}
$$


For the qualitative model, $\beta$ is the parameter giving the probability of an allele dropout (considered as the "basic model") $[10,17]$. Here the evidence profile data $(E)$ are the presence/absence of the alleles. For the quantitative model (EFM), the peak heights (P.H.) of each allele are also considered as part of $E$, and here $\beta$ is the set of following parameters: "Mixture proportions", "P.H. expectation", "P.H. variability", "Degradation slope" (if turned on), "Stutter prop." (if turned on). The MLE approach means that these parameters are selected such that they maximize the probability of observing the evidence profile $E$. The number of contributors, $K$, is estimated using the method based on the qualitative model as presented in the Appendix of Bleka et al. [26]. Here the number of contributors is optimized using ' $\ln L_{d}-K^{\prime}$ as a model selection criterion, initially with $K=1$.

For both the qualitative and the quantitative models, allele frequency data were used to inform the genotype probabilities. Unobserved alleles were assigned with the minimum observed allele frequency ( 0.0018 for GlobalFiler and 5.2e-05 for Fusion 6C frequencies).

\subsubsection{Simple allele comparison}

The first step to compare references against mixture profiles is carried out by applying the MAC. In CS, MAC is given as a percentage of the alleles of the reference that are included in the evidence profile (given as a decimal format). Empty loci are ignored. This method does not use the allele frequencies, information of the peak heights or the detection threshold indicated in the model. The results for all comparisons are present in the panel Match matrix (see Fig S4a in supplement C.1). A user specified "MAC threshold" is utilised to select the candidates for the next comparison step which is based on LR. The default threshold in CS is 0.8 (this corresponds to a $20 \%$ mismatch with the evidence profile), making it potentially possible to detect contributors with a lot of dropouts in lowtemplate evidence profiles. Depending on the application, it is for the user to decide the value of the threshold.

\subsubsection{Comparisons based on LR}

In the second step (which is based on applying the qualitative model), the LR is calculated for each candidate generated from the first step: Here, the number of contributors $(K)$ is estimated for each evidence profile, and then the corresponding candidate reference profiles are specified in turn as the POI (under definition of the $H_{p}$ proposition). The LR results are then listed in the panel Match list (Qual.LR). Here, the "Qual. LR threshold" is specified by the user in order to select the candidates for the next comparison step (step 3) which is based on the quantitative model. In the third step, the LR for each candidate passing the threshold defined from the second step is re-calculated based on the quantitative model; the number of contributors is defined as described for the second step. The LR results are then listed in the panel Match list (Quan.LR); the user specified "Quan. LR threshold" is utilised to indicate the final list of candidates which is provided in the panel Mixtures. See Figs S4 and S5 for the different panels in supplement C.1.

It is also possible for the user to either skip the step which is based on the qualitative model (step 2), or the step which is based on the quantitative model (step 3). Also, the user can, at any time after running step 2, calculate the quantitative based LR for a specific comparison with a user specified proposition set; he/she can condition on a reference (under both $H_{p}$ and $H_{d}$ ) which is given as a match candidate in the Mixtures panel, and specify the number of contributors $K$. This is very useful in order to improve the estimate of the LR when moving from investigative to evaluative reasoning.

\subsubsection{Pre-specified model parameters/configurations}

The models used to calculate the LR requires the user to specify some parameters which are not optimized by the software. For both the qualitative and quantitative models he/she must specify the drop-in probability. For all analyses we used drop-in probability equal to 0.05 . For the quantitative model we used the drop-in peak height parameter $\lambda$ equal to 0.01 , and the detection threshold (AT) 
and kit as specified for the particular dataset. CS also allows the possibility to set several model configurations: Whether to apply degradation or/and stutter model. In our analysis we always used the degradation model but not the stutter model, since this increases computation time, and instead, a stutter filter was applied (see Appendix of Bleka et al. [26] for performance comparison).

The "advanced options" makes it possible to specify the maximum number of contributors (maxK) that can be investigated for either the qualitative/quantitative models. Either three or four contributors were used as defaults and the results were compared to determine the effects on the LR. Also, the number of optimizations used for the quantitative model is configurable (we recommend 4 optimizations).

As opposed to the interpretation software LRmix and EFM, the user of the current CS version (1.4.0) cannot specify the $\theta$-correction in order to take sub-population structure into account [27] - this is fixed to zero in order to reduce computation time. However, the $\theta$ value is typically low and has little effect on the LR. Once candidate matches have been found, evaluation of the evidence will proceed according to laboratory protocols and a weight of evidence will be provided with the desired $\theta$ value (for instance by using EFM).

\subsection{Deconvolution and other functionalities}

In addition to calculating LR, CS is also capable of performing deconvolution based on the quantitative model (see supplement B.2 for mathematical details). Here the marginal probability of an unknown genotype is estimated for each marker and each contributor. CS lets the user define the "Prob-ratio to next" threshold (we used 10 as threshold); such that a genotype for an unknown contributor is deduced when the ratio between the top genotype and the second-top genotype is above this threshold. As a result, suggestive "unknown" profiles are present in the panel Deconvolution (see Fig S3 in supplement C.1). Here, the information about estimated mixture proportion and conditional references, are also present. The user can later extract this profile to the "reference profile" list for further comparisons against other mixture profiles. See supplement B.3 for more details about deconvolution and for an overview of other functionalities in CS.

\subsection{Performance of database search}

To quantify the performance of CS (as a test) we need to summarize false positives, i.e. noncontributors obtaining results above a specified threshold, and false negatives, i.e. contributors with results below a specified threshold.

When searching the large DNA database, we calculated the expected number of false positives to be obtained for our defined allele matching threshold (based on the allele frequencies) [28]. This was calculated for each evidence profiles by using the function exactMACdistr in the R-package euroformix. In addition, we quantified the time taken and the internal memory required to perform different steps in CS (i.e., importing, visualization and comparison of the data). We did this for various database sizes in order to find the technical limits of CS.

\subsection{Comparison with manual investigation}

In order to verify the benefits of CS we compared the time taken for the software to carry out the full analysis with the approximated time taken to carry out the manual investigation by the forensic scientists. The manual investigation was carried out by first visual inspection of the electropherograms and then all alleles in each marker in the evidence samples were compared to the alleles in references samples. 


\subsection{Hardware and R-packages}

All analyses were run on a laptop with Intel Core i7-6600U CPU with $32 \mathrm{~GB}$ internal memory and a $500 \mathrm{~GB}$ internal SSD. To run CS we used the casesolver_1.4.0 package in the $\mathrm{R} \times 643.5 .1$ software. The graphical user interface of CS requires the packages gWidgets2_1.0-7 and gWidgets2tcltk_1.0-6, whereas the packages euroformix_2.1.0 and forensim_4.3 are required for calculation. The package igraph_1.2.2 is used to create the node graph and the package R2HTML_2.3.2 is used to create the final reports (in HTML format).

\section{Results}

\subsection{Analysis of the real crime case}

\subsubsection{Import}

In order to consider samples with sufficient quality we required at least 7 non-empty markers (all types of markers). Sample $\mathbf{1 6 . 0 3}$ was negative with all markers empty whereas samples $\mathbf{0 6 . 0 1}, \mathbf{1 0 . 0 1}$, $16.05,16.13,18.01,26.01$ contained less than 7 markers. These were removed from the analysis. Some samples contained data from very few markers: The samples 19.01, 40.03 and 08.01 (Fig 1) had 8,10 and 14 non-empty autosomal markers, respectively.

\subsubsection{Comparison against single source profiles}

68 of the total 112 evidence samples imported were classified as SS profiles $(K=1)$. In this case, two (related) reference profiles which were the victims, REF1 and REF2, had been sampled (sharing 27 alleles) and many of the SS profiles matched these ( 29 samples matched REF1 and 17 samples matched REF2). CS was also able to assign 22 of the SS profiles as an unknown male profile (Unknown 1), which subsequently matched a known individual who had previously inhabited the flat (REF3). The remaining 44 evidence samples were categorized as mixtures ( $K$ was at least 2 ).

\subsubsection{Comparison against mixtures}

As a starting point for the analysis, $M A C=0.8$ and $L R=1000$ were used as $L R$ thresholds (for both

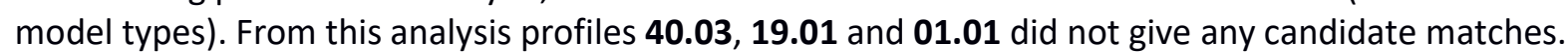
We were able to extract a full major profile, MAJ, from sample 01.01 using the deconvolution function (assuming $K=2$ ). This profile was again compared to all of the other mixtures using the same thresholds. The first step provided a list of 76 comparisons with a MAC above 0.8 (176 comparisons completed within $1 \mathrm{~s}$ ), whereas 73 of these had a qualitative LR above 1000 (completed in $21 \mathrm{~s}$ ). Out of these, 70 comparisons had a quantitative LR above 1000 (completed in $2.8 \mathrm{hrs}$, but restricting the maximum number of contributors to three reduced it to only $10 \mathrm{~min}$ ). Table 1 shows the resulting list after running through all these analyses with the corresponding match tree graph presented in Fig 2.

\begin{tabular}{|r|l|l|l|l|l|l|}
\hline $\begin{array}{l}\text { Sample } \\
\text { Name }\end{array}$ & $\begin{array}{l}\text { \#contr } \\
\text { (by view) }\end{array}$ & References & MixProp (\%) & MAC (\%) & $\begin{array}{l}\text { Qual. LR } \\
\text { (log10) }\end{array}$ & $\begin{array}{l}\text { Quan. LR } \\
\text { (log10) }\end{array}$ \\
\hline 01.01 & $2(1 ?)$ & MAJ & 91 & 100 & 16.5 & 29.4 \\
\hline 03.01 & 2 & REF2/REF1 & $72 / 28$ & $100 / 100$ & $18.5 / 18.8$ & $29.4 / 18.7$ \\
\hline 03.02 & 2 & REF2/REF1 & $79 / 21$ & $100 / 98$ & $17.3 / 17.2$ & $29.5 / 13.0$ \\
\hline 04.01 & $3(4)$ & REF1/REF2/REF3/MAJ & - & $100 / 100 / 100 / 98$ & $10.3 / 9.9 / 7.6 / 8.3$ & $28.3 / 16.2 / 13.5 / 11.6$ \\
(maxK=3,4) & $4(4)$ & REF1/REF2/REF3/MAJ & $47 / 23 / 21 / 8.7$ & $100 / 100 / 100 / 98$ & $10.3 / 9.9 / 7.6 / 8.3$ & $28.1 / 16.2 / 13.4 / 10.8$ \\
\hline 05.01 & $2(1)$ & REF3 & 87 & 100 & 16.1 & 20.7 \\
\hline
\end{tabular}




\begin{tabular}{|c|c|c|c|c|c|c|}
\hline 08.01 & 2 & REF2 & 79 & 100 & 8.6 & 13.6 \\
\hline 13.01 & 2 & REF1/REF3 & $85 / 15$ & $100 / 98$ & $17.1 / 11.9$ & 30.9/19.9 \\
\hline 13.02 & $2(1)$ & REF1 & 89 & 100 & 14.6 & 21.6 \\
\hline 14.01 & $2(1)$ & REF3 & 98 & 100 & 16.2 & 26.2 \\
\hline 16.01 & $2(1)$ & REF3 & 95 & 100 & 15.8 & 26.2 \\
\hline 16.02 & 2 & REF3/REF1 & $88 / 12$ & $100 / 83$ & $14.7 / 6.0$ & 26.1/11.7 \\
\hline $16.03 . R$ & $2(1)$ & REF3 & 98 & 100 & 16.2 & 26.2 \\
\hline 16.09 & 2 & REF3 & 84 & 100 & 15.6 & 25.9 \\
\hline 16.11 & 2 & REF1/REF3 & $43 / 57$ & $100 / 100$ & $17.0 / 12.6$ & $18.2 / 15.3$ \\
\hline 16.11.R & 3 & REF3/REF1/ REF2 & $45 / 37 / 17$ & $100 / 100 / 100$ & 10.2/13.3/12.6 & $13.5 / 18.7 / 12.7$ \\
\hline 16.12 & $2(1)$ & REF1 & 88 & 100 & 18.7 & 27.6 \\
\hline 17.01 & 2 & REF3 & 83 & 100 & 15.4 & 22.4 \\
\hline 20.01 & $2(1)$ & REF3 & 93 & 0.97 & 11.8 & 20.0 \\
\hline 21.02 & $2(1)$ & REF3 & 97 & 100 & 16.1 & 26.2 \\
\hline 21.03 & 3 & REF3/REF1/REF2 & $62 / 25 / 13$ & $100 / 100 / 98$ & $10.3 / 13.3 / 10.0$ & $18.3 / 17.6 / 10.9$ \\
\hline 26.01.R & $2(3 ?)$ & REF1/REF2 & $80 / 20$ & $100 / 100$ & $18.5 / 18.2$ & 30.9/10.8 \\
\hline 30.01 & 1 & REF1 & 100 & 100 & 27.3 & 30.8 \\
\hline $30.01 . R$ & $3(2 ?)$ & REF1/REF3/REF2 & $65 / 31 / 4.4$ & $100 / 100 / 90$ & $13.2 / 10.1 / 6.7$ & 26.5/16.9/6.5 \\
\hline 37.01 & 1 & REF1 & 100 & 100 & 27.0 & 30.9 \\
\hline 37.03 & 2 & REF1/REF2 & $51 / 49$ & $100 / 100$ & $18.7 / 18.3$ & $25.3 / 23.7$ \\
\hline 38.06 & $2(1)$ & REF1/REF2 & $89 / 11$ & $100 / 86$ & $18.4 / 9.7$ & $30.9 / 4.7$ \\
\hline 38.15 & $2(1)$ & REF1 & 97 & 100 & 19.2 & 30.9 \\
\hline 39.01 & 2 & REF2/REF1 & $76 / 24$ & $100 / 95$ & $18.3 / 16.6$ & $28.8 / 16.2$ \\
\hline 39.04 & 2 & REF2 & 91 & 100 & 17.9 & 29.6 \\
\hline 39.05 & $2(1)$ & REF2 & 91 & 100 & 17.6 & 29.6 \\
\hline 39.06 & 1 & REF2 & 100 & 100 & 26.4 & 29.6 \\
\hline 39.07 & 2 & REF1/REF2 & $72 / 28$ & $100 / 100$ & $18.8 / 18.5$ & $30.5 / 18.8$ \\
\hline 39.08 & $2(1)$ & REF2/REF1 & $91 / 8.8$ & $100 / 86$ & $18.4 / 12.5$ & $29.6 / 4.7$ \\
\hline 39.09 & 2 & REF1 & 96 & 100 & 18.2 & 30.9 \\
\hline 40.01 & $2(1)$ & REF2 & 92 & 100 & 17.6 & 29.6 \\
\hline 40.02 & $2(1)$ & REF2 & 97 & 100 & 18.9 & 29.5 \\
\hline 40.04 & $2(1)$ & REF2 & 95 & 100 & 18.9 & 29.5 \\
\hline 40.05 & 3 & REF3/REF1/REF2 & $62 / 22 / 16$ & $100 / 100 / 98$ & $10.4 / 13.5 / 11.5$ & $19.8 / 17.8 / 12.2$ \\
\hline 40.05.R & 3 & REF3/REF2/REF1 & $45 / 33 / 22$ & $100 / 100 / 100$ & 10.4/12.9/13.4 & $12.9 / 16.0 / 16.0$ \\
\hline 41.01 & 2 & REF2/REF1 & $88 / 12$ & $100 / 90$ & $16.0 / 10.8$ & $29.6 / 4.0$ \\
\hline 41.01.R & 3 & REF2/REF3/REF1 & 70/14/16 & $100 / 100 / 100$ & $12.5 / 10.2 / 13.2$ & 29.5/15.2/10.8 \\
\hline 45.01 & 2 & REF1/REF2 & $56 / 44$ & $100 / 100$ & $18.7 / 18.3$ & $26.9 / 22.2$ \\
\hline
\end{tabular}

Table 1: The table shows the match candidates from CS based on the three-step search strategy with $\mathrm{MAC}=0.8$ and $\mathrm{LR}=1000$ as thresholds. The column "\#contr" indicates the number of contributors estimated by CS, with manual inspected given in parenthesis. The question mark indicate that the manual inspection is highly uncertain. The mixture proportion is estimated by conditioning on all matching references (at the same time) and "\#contr" as the number of contributors. Candidates with 
LR values between $1 \mathrm{e} 3$ and $1 \mathrm{e} 6$ are indicated in gray. Sample 04.01 was evaluated with both 3 and 4

\section{Matches for RealCase1}

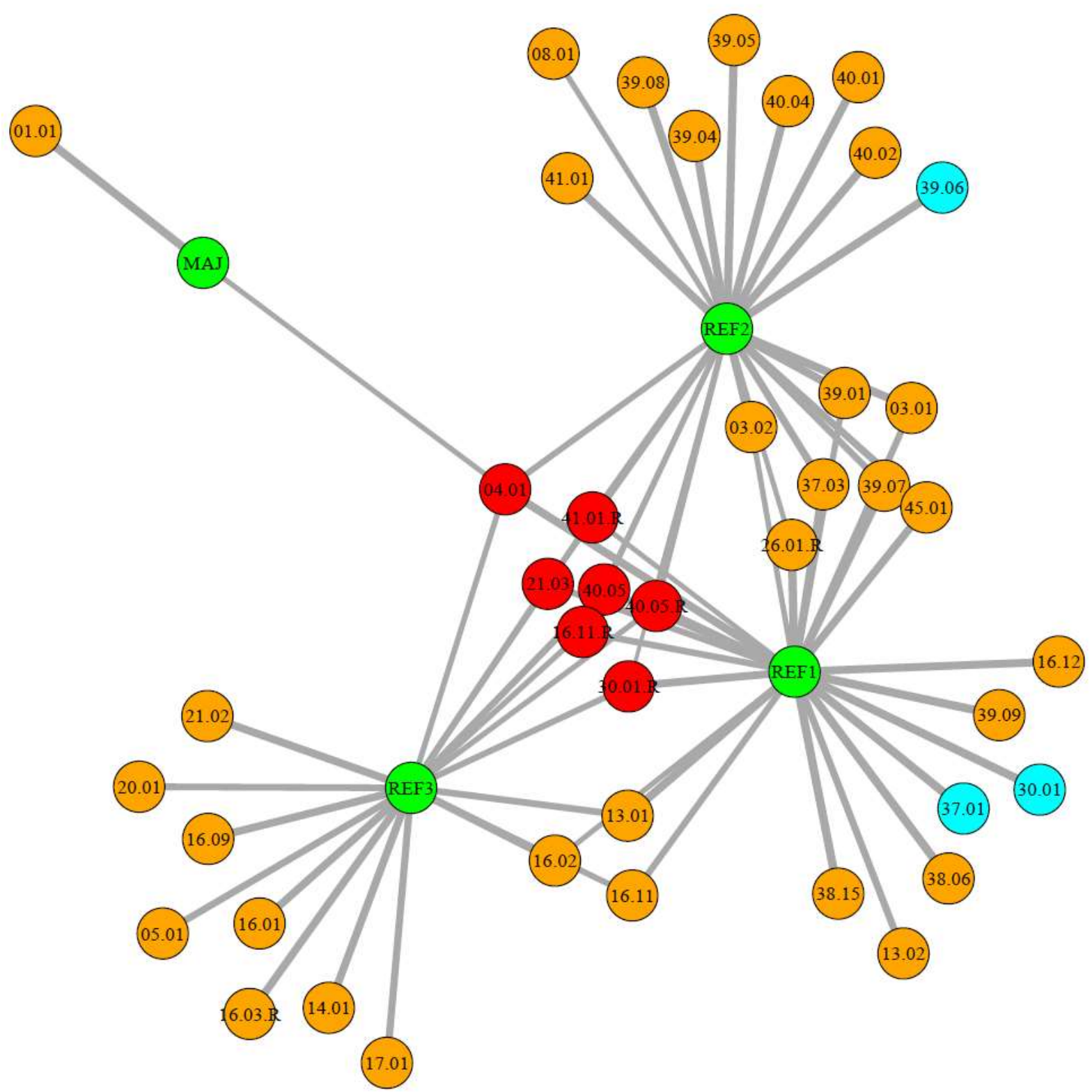

Fig 2: The figure shows the graph plot generated from the final match list (quantitative log $10 \mathrm{LR}=6$ used as threshold), where the thickness of the edges and the distance between the nodes are inversely proportional to the quantitative based LR values (on log10 scale). Green nodes are reference profiles, cyan nodes are single source profiles, whereas the orange and red nodes are mixtures; orange nodes are mixtures with 2 contributors, and red nodes are mixtures with more than 2 contributors (estimated).

\subsubsection{Further inspections}

CS has the functionality of (suboptimal) testing for relatedness by comparing the number of shared alleles between references that are identical by state (IBS). For this, CS can calculate the distribution 
of IBS under the assumption that references are unrelated (see table S3 in supplement C.2). This distribution is useful for testing whether two references are related or not. In the real case example we calculated IBS between 4 references ( 6 pairwise comparisons), and found that $I B S \geq 20$ was a threshold that gave a $p$-value less than $5 \%$ against of being unrelated $(6 * 3.4 \mathrm{e}-3=0.02)$. REF1 shared 27 alleles with REF2 ( $p$-value=7.3e-7), whereas MAJ shared 24 alleles with REF3 ( $p$-value=1.2e-4). When there is limited information in a case, information on potential relatedness would be useful to investigations.

In addition to the standard search strategy we considered a more thorough analysis to discover potential false negatives or positives (see supplement C. 2 for more details about comparisons that were carried out). Here we lowered the MAC threshold to 0.7 and the qualitative based LR threshold to 1 , and then applied the quantitative model where we conditioned on presumed matching profiles. Consequentially, we obtained the following (updated) results in about 1 hour of work using CS:

Potential false positives:

- 30.01.R - REF2 (MAC=0.9):

- Conditioning on REF1 with $K=3$ contributors, reduced $L R$ from $10^{\wedge} 6.4$ to $10^{\wedge}(-0.2)$

- Conditioning on REF1/REF3 with $K=3$ contributors, reduced LR further to $10^{\wedge}(-1.7)$

- 38.06- REF2 (MAC=0.86):

- Conditioning on REF1 with $K=2$ contributors, reduced LR from $10^{\wedge} 4.6$ to $10^{\wedge}(-4.1)$

- Conditioning on REF1 with $K=3$ contributors, reduced $L R$ from $10^{\wedge} 4.6$ to $10^{\wedge}(1.7)$

Potential false negatives:

- $\quad 39.04-\operatorname{REF} 3$ (MAC $=0.76$ ): Conditioning on REF2 with $K=2$ contributors, returned $L R=10^{\wedge}(9.4)$

- 41.01 - REF3 ( $M A C=0.79$ ): Conditioning on REF2 with $K=2$ contributors, returned $L R=10^{\wedge}(8.7)$

- 40.03 - REF3 (MAC=0.75): Conditioning on none with $K=2$ contributors, returned $L R=10^{\wedge}(4.0)$

REF3 has allele 9 in marker DYS391, which was also obtained for all the three mixtures, increasing the support that REF3 could be a contributor (however this was not taken into account in the LR calculation).

\subsubsection{Comparison with manual investigation}

It was very difficult to calculate the time spent on the manual work (revision of data and comparison) since this was carried out as the samples are analyzed. A rough approximation on the time taken was 8 hours: The time taken for the comparison depended on the complexity of the evidence profiles. In general, when these are SS profiles with good quality, the comparison is easy. Comparison with higher order mixtures took much longer time and sometimes there were no clear conclusions. In addition, as some profiles come from related individuals (two of the references shared many alleles), the probability of making a mistake was high.

\subsection{Managing and searching of a large fictive national DNA database}

One million randomly generated reference profiles were imported into CS along with the real case data. We monitored the system requirement in terms of speed, memory and storage (see table S7 in supplement C.3 for detailed information of different database sizes). The time taken to simulate, import and visualize the data was about $14 \mathrm{~min}$, with a high internal memory requirement of 13.4 $\mathrm{GB}$. Storing the project required a file of $50 \mathrm{MB}$ which could be imported in about $10 \mathrm{~min}$. The time taken to compare all 1 mill. references (incl. case refs.) against the 44 classified mixtures with the MAC method (44 mill. comparisons in total), and generating the results in a huge table, was $34 \mathrm{~min}$ and required $12.5 \mathrm{~GB}$. Of these, 56,521 comparisons satisfied the threshold of MAC $=0.8$. We next calculated the qualitative LR for all candidates (completed in almost 8 hours). A total of 302 of the comparisons satisfied a qualitative LR of at least 1000. Calculating the quantitative LR for these 
remaining candidates took $16 \mathrm{~min} / 4 \mathrm{hrs}$ ( $\operatorname{maxK}=3 / 4$ ), and resulted in 210/190 match candidates with LR above 1000 ( $\operatorname{maxK}=3 / 4$ ) and 68 with LR above 1e6. Table 2 gives an overview of the number of generated references found after applying the "threshold test" in each of the comparison steps in CS. The presumed four-person mixture profile 04.01 yielded the most false positives due to the high number of observed alleles, whereas the qualitative LR method removed most of these with a threshold LR=1000.

In the next step, the quantitative model reduced the number of candidates further; however, many candidates still had a LR above 1000. For the presumed four-person mixture profile $\mathbf{0 4 . 0 1}$ the number of false positives was increased from 7 to 27 when changing from three to four contributors in the quantitative model; here the LR of the top ranked generated reference was increased. The largest false positive quantitative based LR for a randomly generated reference was $\log 10 \mathrm{LR}=6.1$ (MAC=0.94), obtained for the low quality sample 19.01. This was the only comparison with a $L R$ above 1 mill. Hence all the comparison results for the known references in the previous section (Table 1) obtained the highest rankings when a LR threshold of 1 mill. was used.

\begin{tabular}{|l|l|l|l|l|l|l|l|}
\hline Sample Name & Expected & MAC=0.8 & $\begin{array}{l}\text { Qual. } \\
\text { LR=1000 }\end{array}$ & $\begin{array}{l}\text { Max Qual. LR } \\
\text { (log10) w/MAC }\end{array}$ & $\begin{array}{l}\text { Quan. } \\
\text { LR=1000 }\end{array}$ & $\begin{array}{l}\text { Quan. } \\
\text { LR=1e6 }\end{array}$ & $\begin{array}{l}\text { Max Quan.LR } \\
\text { (log10) w/MAC }\end{array}$ \\
\hline 04.01 (maxK=3/4) & 44567 & 44397 & 41 & $5.3(0.95)$ & $7 / 27$ & $0 / 0$ & $4.8(0.95) / 5.9(0.95)$ \\
\hline $16.11 . R$ & 2691 & 2665 & 12 & $4.6(0.90)$ & 2 & 0 & $4.8(0.88)$ \\
\hline $41.01 . R$ & 2456 & 2466 & 10 & $5.9(0.93)$ & 6 & 0 & $5.0(0.86)$ \\
\hline $30.01 . R$ & 1616 & 1639 & 24 & $4.8(0.88)$ & 23 & 0 & $4.5(0.86)$ \\
\hline 21.03 & 1183 & 1200 & 21 & $5.3(0.90)$ & 13 & 0 & $4.7(0.86)$ \\
\hline 19.01 & 1170 & 1190 & 35 & $4.5(0.94)$ & 32 & 1 & $6.1(0.94)$ \\
\hline 40.05 & 1120 & 1182 & 17 & $5.3(0.90)$ & 8 & 0 & $4.6(0.86)$ \\
\hline $40.05 . R$ & 1073 & 1100 & 17 & $5.3(0.90)$ & 6 & 0 & $3.9(0.90)$ \\
\hline 16.11 & 226 & 251 & 0 & $-0.4(0.81)$ & - & - & - \\
\hline 40.03 & 114 & 94 & 24 & $4.7(0.85)$ & 22 & 0 & $5.5(0.85)$ \\
\hline 13.01 & 83 & 82 & 0 & $2.4(0.81)$ & 0 & 0 & $5.3(0.83)$ \\
\hline 16.02 & 72 & 65 & 0 & $2.8(0.81)$ & - & - & - \\
\hline 08.01 & 43 & 57 & 26 & $4.6(0.82)$ & 21 & 0 & $4.7(0.82)$ \\
\hline 41.01 & 35 & 41 & 1 & $3.6(0.81)$ & 0 & - & $-4.2(0.81)$ \\
\hline 39.04 & 11 & 13 & 1 & $3.4(0.81)$ & 0 & - & $-2.0(0.81)$ \\
\hline $38.06 / 16.09 / 39.05$ & $1.4 / 0.6 / 0.4$ & All 1 & All 0 & $2.0 / 1.0 / 2.5$ & & - & - \\
\hline & & & & $(a l l 0.81)$ & & & \\
\hline
\end{tabular}

Table 2: The table gives an overview of the observed number of generated references (out of 1 mill. total) found after applying the filter in each of the comparison steps in CS, for each evidence profile. "Expected" is the expected number of references which are expected to satisfy the MAC $=0.8$ threshold. ". Here, only evidences with at least one generated reference candidate, are presented. For sample 04.01 we evaluated both for $\mathrm{K}=3$ and $\mathrm{K}=4$ number of contributors for the quantitative model. The last column presents the maximum observed LR for the generated reference profile with the observed MAC presented in parentheses.

\subsection{Analysis of the Fusion $6 C$ validation study}

In this section we show the results of using CS on data from the validation study (25 mixtures and 14 references) where the ground truth is known (either the reference is a contributor or a noncontributor). Here, a number of false positive and negative results were observed out of 350 
comparisons - the number was dependent upon the values of the "MAC threshold", "Qual.LR threshold" and "Quan.LR threshold". The results are presented in Table 3. False positives were only observed using the MAC method (comparison S00_B03 - R15 were 0.91 was the highest observed), whereas both the qualitative/quantitative based methods eliminated all instances. The highest qualitative based LR for a non-contributor was $\log 10 \mathrm{LR}=-2.3$ (comparison S01_B01-R23).

Table 3 shows that different threshold settings influence the number of false negatives, but has little effect on the number of false positives. The best strategy can be defined as the one which minimizes numbers of false positives and negatives - using "MAC threshold" $=0.7$ and "Qual. $L R$ threshold" $=1$ without using the quantitative based method (see Fig S2 in the supplement A.2 for a graph plot based on the final match list). Here only one false negative was observed: S02_B01 - R7, where the reference had MAC $=0.74$ ( 12 dropouts) and the qualitative $\log 10 \mathrm{LR}=-2.2(K=2)$. The number of contributors for this evidence profile had been underestimated. For the same sample and for the same reason, the quantitative model additionally returned S02_B01 - R15 as a false negative having $M A C=0.85$ ( 7 dropouts) and quantitative $\log 10 \mathrm{LR}=-4.7(K=2)$. When the true number of contributors was conditioned on $(K=3)$, the quantitative based method gave $\log 10 \mathrm{LR}=12.6$ for $\mathrm{R} 7$ and $\log 10 \mathrm{LR}=4.2$ for R15.

The quantitative based method returned four false negatives with four-person mixtures, where the number of contributors were only considered as three: The comparisons S00_B01 - R5/R10 and S00_B04-R5/R10. CS estimated these mixtures as four contributors but there was, by default, an upper limit for the quantitative model of three contributors imposed, in order to speed-up calculations. Setting four contributors as a maximum (instead of three) solved this issue.

\begin{tabular}{|l|l|l|l|l|l|l|}
\hline MAC & Qual.LR & $\begin{array}{l}\text { Quan.LR } \\
(\operatorname{maxK})\end{array}$ & Time & $\begin{array}{l}\text { \#Comp. } \\
\text { qual/quan }\end{array}$ & $\begin{array}{l}\text { False } \\
\text { Positives }\end{array}$ & $\begin{array}{l}\text { False } \\
\text { Negatives }\end{array}$ \\
\hline 0.8 & 1000 & - & $2 \mathrm{~min}$ & $82 /-$ & $17 / 0 /-$ & $5 / 8 /-$ \\
\hline 0.8 & 1 & - & $2 \mathrm{~min}$ & $82 /-$ & $17 / 0 /-$ & $5 / 5 /-$ \\
\hline 0.8 & 1 & $1000(3)$ & $26 \mathrm{~min}$ & $82 / 65$ & $17 / 0 / 0$ & $5 / 5 / 10$ \\
\hline 0.8 & 1 & $1000(4)$ & $12.5 \mathrm{hrs}$ & $82 / 65$ & $17 / 0 / 0$ & $5 / 5 / 6$ \\
\hline 0.8 & 1 & $1(3)$ & $26 \mathrm{~min}$ & $82 / 65$ & $17 / 0 / 0$ & $5 / 5 / 9$ \\
\hline 0.8 & 1 & $1(4)$ & $12.5 \mathrm{hrs}$ & $82 / 65$ & $17 / 0 / 0$ & $5 / 5 / 6$ \\
\hline 0.7 & 1000 & - & $2 \mathrm{~min}$ & $124 /-$ & $54 / 0 /-$ & $0 / 7 /-$ \\
\hline 0.7 & 1 & - & $2 \mathrm{~min}$ & $124 /-$ & $54 / 0 /-$ & $0 / 1 /-$ \\
\hline 0.7 & 1 & $1000(3)$ & $26 \mathrm{~min}$ & $124 / 69$ & $54 / 0 / 0$ & $0 / 1 / 6$ \\
\hline 0.7 & 1 & $1000(4)$ & $12.5 \mathrm{hrs}$ & $124 / 69$ & $54 / 0 / 0$ & $0 / 1 / 2$ \\
\hline 0.7 & 1 & $1(3)$ & $26 \mathrm{~min}$ & $124 / 69$ & $54 / 0 / 0$ & $0 / 1 / 5$ \\
\hline 0.7 & 1 & $1(4)$ & $12.5 \mathrm{hrs}$ & $124 / 69$ & $54 / 0 / 0$ & $0 / 1 / 2$ \\
\hline
\end{tabular}

Table 3: The table shows the number of false positives/negatives for different sets of thresholds (for each row). MAC is the threshold of proportion of matching alleles of references whereas "Qual.LR" and "Quan.LR" are the LR thresholds for the qualitative and quantitative based methods, respectively. "maxK" is the maximum number of contributors allowed. "\#Comp." is the number of comparisons when performing either the qualitative based method or quantitative based method. The format $x / y / z$ means that $x, y$ and $z$ is the number of false positives/negatives after sequentially applying the "MAC threshold" ( $\mathrm{x}$ ), the "Qual.LR threshold" (y) and the "Quan.LR threshold" (z). "-" means that the method was not applied.

\section{Discussion}

\subsection{The need for digital tools in forensic genetics}

The rapid change and use of DNA technology requires new analytical software to be developed in order to deal with vast amount of information generated. Traditional DNA analyses are often based 
on manual comparisons of alleles, together with peak heights, using pen and paper. As the number of markers increase, such work requires a lot of resources and transcription errors are a particular concern. The increased sensitivity of DNA typing has also led to the generation of more mixture profiles, and analysing these together with a large number of other profiles in complex cases is required by routine caseworkers.

In this paper we have introduced an exploratory investigative tool to filter large sets of data in order to discover candidate matches (potential matches between reference profiles and evidence profiles), either single source profiles or mixtures. We provide a demonstration of a challenging case, and we also show the benefit of being able to perform more thorough analyses (LR calculation, deconvolution or simple relatedness testing) where hypotheses can be specified more carefully, without exporting to other software.

\subsection{Improving the efficiency of complex investigations}

With complex investigations there are often no suspect(s). Under these circumstances, investigators will usually resort to a search of the national DNA database in order to discover potential candidates. Whereas this is quite a straightforward process for single, well represented DNA profiles, when the evidence profiles are mixtures a different approach is needed. Most national DNA databases are geared to work with single source profiles. If a mixture is obtained then deconvolution may be carried out in order to extract a candidate reference to search with, but this procedure is problematic because it is realistically restricted to clear major contributors; if the person of interest is a minor contributor, the approach does not work because masking and drop-out means that there can be no definitive reference sample to search with. As a result, reliance upon deconvolution is inefficient as many crime-stains cannot be analysed. Consequently, many cases remain unsolved.

The likelihood ratio approach employed by probabilistic models is a preferable way forward because there is no restriction on profiles that can be searched (no deconvolution) so that minor contributors can be investigated - there is no need for a clear major contributor to be the person of interest. Such an approach is employed by SmartRank [18]. This is a rapid search engine that employs the qualitative model of Haned et al. [17]. However, quantitative models are more informative because they take account of the peak height information in the evidence profile. But the downside is that the computational requirements are much greater. Consequently, this may act as a limitation on the number of reference samples that may be searched. In order to solve this problem, we have devised a three-step test. Clearly, there is no point in carry out extensive computations on samples where only a few alleles match. Therefore, these can be quickly filtered by carrying out simple allele matching and eliminating those references that fall below a threshold. The remainder can be searched by the qualitative model, and the final list refined by the quantitative model.

The investigative procedure is outlined in Fig 3. A list of reference profiles to search may arise from staff elimination databases (contamination search); mass screens of local populations; national DNA databases; or persons of interest highlighted as a result of other investigations. The purpose of the lab is to compare the reference profiles against the relevant evidence profile(s) to provide a ranked list of candidates. This list is forwarded to the police who carry out further investigations. The list of candidates may be restricted according to criteria such as age or geographic location [29]. If phenotyping information is available e.g. geographic ancestry or hair/eye colour, then this can also be used to reduce the size of candidate lists [30], noting that no test is $100 \%$ definitive hence there is a risk of false negatives.

Either all candidates are eliminated, or one is identified as a suspect who is carried forward to the prosecuting authorities for evaluation based on additional evidence in the case. If all are eliminated, 
the police contact the lab and request an extension to the candidate list, either including references with lower ranking, or by considering a new set of references based upon new criteria.

When there is sufficient non-DNA evidence in the case to proceed with the prosecution, then the scientist switches to evaluative mode as reviewed by Gill et al. [31]. Once in the evaluative mode the strength of evidence is considered using refined propositions that are posited by the relevant authorities [32], and with suitable ' $\theta$-correction' (the current version of CS does not consider ' $\theta$ correction') and other settings discussed in Bleka et al. [8]. Importantly, CS can export the selected DNA profiles directly to EFM, or to text files for other software users, so that there is no method restriction for the evaluative phase to take place.

The idea of investigative searches is to prioritise police resources where there is limited information in the case. If the DNA evidence profile is non-complex and well represented at $16+$ loci then there is usually no issue with searching very large reference databases of several million individuals. The risks of missing someone are low. However, when the profile is a complex mixture of three or more persons then it is inevitable that there are many false positive matches in the candidate list. In order to carry out an investigation, this list is ranked according to the likelihood ratio, and the police will investigate the individuals in this ranked order. In order to forward a person to the evaluative stage, it is necessary to have additional evidence e.g. eye witness, fibres. It is therefore a time-consuming process. For example, if no candidate is found after the first one hundred ${ }^{1}$ comparisons, then there is an option to extend the list to the next one hundred, and so-on. This process is dictated entirely by the police investigator and it is ultimately dependent upon the resources and time available to investigate a particular crime. There will be more resources for a serial killing than for a petty burglary. In addition, rather than search entire databases, subsets of databases may be prioritized based on, for example, geographical location, age and sex of proposed offender; previous convictions of offenders with a known modus operandi etc. These are examples of criteria that may be used to prioritise investigations without the need to search entire databases.

\footnotetext{
${ }^{1}$ Or some other number decided by the investigating authorities
} 


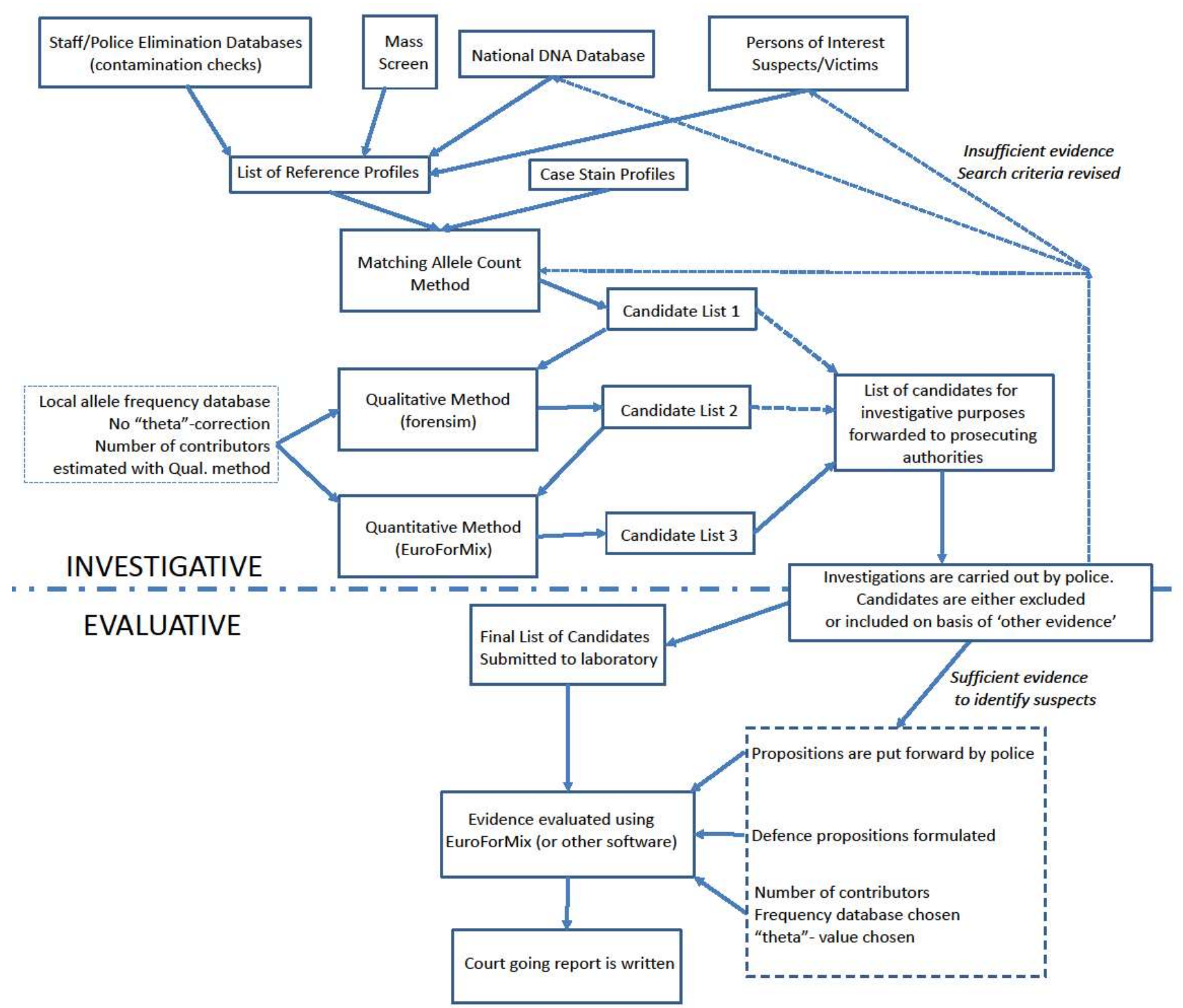

Fig 3: Reference profiles are collected (part of case circumstances or databases) and compared to evidence profiles. A three-step procedure identifies 'matches' which are forwarded to prosecuting authorities for further investigation. Either the candidates are all eliminated, in which case a more relaxed or focused search may be requested, or one or more candidates become suspects and the lab moves from investigative to evaluative mode, in considering the evidence in relation to propositions put forward by the authorities and the case circumstances. Dotted arrow lines are optional.

\subsection{Why use a stepwise strategy to extract candidate matches?}

The LR method is preferred to the MAC when mixtures are compared to large DNA reference databases [25]. Studies show that a quantitative model, such as EFM, outperforms the qualitative model $[26,33]$. However, as illustrated in this paper, the time taken to perform analyses with EFM is a limitation, hence the step-wise approach was introduced. An argument for using MAC as a first step is consistent with many labs that have introduced an upper threshold on the number of mismatches allowed before a result can be reported. For example, if, the analytical procedure accepts a maximum of 4 dropouts, such a rule would be easy to apply in CS with the added benefit of speeding up comparisons (as opposed to not using this threshold). 
CS makes use of the qualitative model since it is very fast and performs well (also implemented in SmartRank [18]). We have implemented the MLE approach for improved performance [26]. For the Fusion $6 \mathrm{C}$ validation study we found that this approach performed well by giving a high LR for contributors and a low LR for non-contributors (except for the high dropout samples where the number of contributors were underestimated). However, as illustrated for the real case example, this model can sometimes give misleading LR values since it does not use the peak height model - this would typically happen when searching large DNA databases or considering relatives, as illustrated in this paper and by others [26]. Consequently, we recommend that the three-step strategy is normally applied (Fig 3). This paper (along with a previous study [21]) shows that this is accomplished within a reasonable time period.

\subsection{The performance of searching large national DNA databases}

In order to test the limitations of CS, we tried different sizes of reference databases which were based on 24 loci (also included non-autosomal markers). For 1 mill. generated references, in addition to the profiles in the real case example, the memory required was $13 \mathrm{~GB}$. It was not possible to carry out computations with an $8 \mathrm{~GB}$ platform. Once all the data were imported in $\mathrm{CS}$, the total time taken for CS to compare and visualize the results of comparing more than 1 mill. references against 44 mixtures was $35 \mathrm{~min}+$ "additional". Here, "additional" refers to the strategy used after the MAC comparison. As a result, we found that the qualitative/quantitative based methods were important to apply in order to reduce the very high number of false positives: The MAC $=0.8$ threshold returned 56,445 (generated) candidates, where the higher order mixture sample 04.01 constituted 44,397 of these. Sequentially using the qualitative method with threshold $L R=1000$ recovered 229 (generated) candidates. Last, when the quantitative model was applied, an LR threshold of 1000 was suitable to obtain a manageable list of 140/160 (generated) candidates (maxK=3/4). However, increasing it to 1 mill removed almost all (generated) references from the match list, except of one instance which had $\log 10 \mathrm{LR}=6.1$. This observation is entirely in keeping with theory by Gill et al. (2014) [28] who stated: "How often a $\mathrm{LR}=x$ or more occurs is accommodated by $P(L R>x \mid H d)<1 / x$, so we never expect more than $N / x$ chance matches, where $N$ is the size of the database. The number of noncontributors with a greater LR than $x$, in a database of size $N$, is $N^{*} P(L R>x \mid H d)$ and it is crucially dependant on $N$." Hence searching against a large number of reference samples with low LRs as thresholds causes an investigative limitation because of the increase of false positive results. Still, the number of false positives observed in our demonstration were much less than the expected upper boundary from such a search, i.e., 'number of samples'*'database size'/'LR threshold' $[5,28]$. This would be 44,000 with a threshold of $L R=1000$ and 44 with $L R=1 e 6$.

Regarding large DNA databases, CS is very memory demanding. The bottleneck comes down to the visualization of large matrices in the graphical user interface. We recommend using the non-graphical software dnamatch2 [21] as a memory efficient alternative (it is based on the same methods used in CS). Another alternative is to use EFM or SmartRank [18] for searching one evidence profile (also replicates) at a time in very large DNA databases for a specified set of propositions.

\subsection{The flexibility of CS}

An important aspect of CS is that it is very easy to customise: The user can provide different model settings and search strategy thresholds, and even tailor the type of data that can be imported into the software. CS also has the possibility to create simple HTML reports, with the limitation that it does not directly produce reports in pdf format. Here, the user can select what part of the data or results that should be exported to the report. 
CS makes it possible to define a lab-specific "importData" function. This means that there are no rules to describe the format of DNA profiles used as input, so long as the function returns a valid format which is recognizable by CS. This enables a general framework to import data into CS, limited by the R language and its supporting R-packages. This flexibility of approach means that it can be integrated into laboratory systems that have different data-formats to that used by us.

Another possibility, which is currently implemented, would be to use CS to analyze next generation sequencing based data where one may want to consider the "longest uninterrupted sequence (LUS)" format (together with the ForenSeq kit, for instance) as presented in Just and Irwin (2018) [34].

A tutorial for the software is available at http://www.euroformix.com/casesolver.

\subsection{The validation status of CS}

The software is 'investigative' rather than 'evaluative'. The aim is to capture as many true 'matches' as possible, minimizing the number of false negatives and false positives (section 4.3). CS is an improvement on standard database screening that relies only on deconvolution and is also an improvement on qualitative screening used by SmartRank. Defining the performance of software in terms of false positives and negatives is an important part of validation and provides a benchmark statement of the performance to compare future improvements, i.e., validation is a continuous process. There will be limitations, particularly when the person of interest is a contributor with very low level because the corresponding LRs will be low and below threshold (section 4.3). The system is designed to be exploratory and there is flexibility for the user. After 'matches' have been found, the scientist enters the evaluative phase of the analysis which forms the basis of the court report - this means that the LR can be recalculated with EFM or any other probabilistic software using specific frequency databases, $\theta$-correction, number of contributors etc. as shown in Fig. 3 and this is covered by separate validation studies reported elsewhere (e.g. $[26,35,36])$.

Supplement $D$ describes program checking carried out using $R$ scripts that are available at the webpage www.euroformix/evaluations in a file named 'NumEval 2.1.0'.

Since November 2017, CS has been actively used in casework at the Department of Forensic Sciences (Oslo University Hospital) in Norway. Here, CS has been integrated into the data system and work flow in order to allow seamless transfer of data. We have found CS to be an important tool to support the findings of the reporting scientists, and to discover manual typing errors or artefacts from an earlier stage in the analysis process. There is a lot of flexibility in the software so that it can be utilized for specific purposes. Consequently, it is necessary for labs to carry out in-house validation but this is true for any software.

\subsection{Future improvement}

The estimation of the number of contributors was conducted by using the maximum likelihood estimation of the qualitative model with a penalty term, as which was introduced in Bleka et al. [26]. From the examples in the paper, we found some situations where the number of contributors was underestimated, leading to false negatives. We hope to further improve the method in a future update.

The method for relatedness testing in CS was only based on simple allele sharing (IBS). In a future update the LR for hypothesized relationships could also be calculated to further improve the test.

More sophisticated theory can be developed to identify whether contributors in two separate mixtures are the same. By generalizing the traditional methodology it is possible to calculate a LR score for the comparison of proposition "Two mixtures share a common contributor" versus "Two 
mixtures do not share any contributors"[37]. Hence this could be applied to make investigative leads about common unknown contributors of complex mixtures that are otherwise difficult to resolve.

\section{Conclusion}

We have demonstrated that CaseSolver is an efficient graphical expert tool to produce a fast overview in forensic cases with many single source and references profiles together with complicated mixtures. It is ideal when there are many pairwise comparisons needed (typical for screening large databases). This shows that applying such tool in DNA-labs would dramatically improve the efficiency of profile comparisons. However, it is important to separate the investigative from the evaluative process of interpretation - CS is an investigative tool used to support the reporting scientists, to discover potential suspects whom may be put forward for evaluative purposes. Evaluation can be carried out with respect to any probabilistic software (i.e. it is not limited to EuroForMix).

\section{Acknowledgement}

We want to thank the laboratory, caseworkers and scientists at Oslo University Hospital for providing the Fusion $6 \mathrm{C}$ validation study data, and for testing the software with real case applications. Also many thanks to Santiago Hernandis for assisting us with the crime scene map. We thank the reviewers for improving the paper.

\section{Conflict of interest}

The authors declare that they have no conflict of interest.

\section{Appendix}

\section{Supplementary material}

\section{References}

[1] P. Gill, A. Urquhart, E. Millican, N. Oldroyd, S. Watson, R. Sparkes, C.P. Kimpton, A new method of STR interpretation using inferential logic--development of a criminal intelligence database, International journal of legal medicine 109(1) (1996) 14-22.

[2] C. Kimpton, D. Fisher, S. Watson, M. Adams, A. Urquhart, J. Lygo, P. Gill, Evaluation of an automated DNA profiling system employing multiplex amplification of four tetrameric STR loci, International journal of legal medicine 106(6) (1994) 302-11.

[3] M.J. Ludeman, C. Zhong, J.J. Mulero, R.E. Lagacé, L.K. Hennessy, M.L. Short, D.Y. Wang, Developmental validation of GlobalFiler ${ }^{\mathrm{TM}}$ PCR amplification kit: a 6-dye multiplex assay designed for amplification of casework samples, International journal of legal medicine 132(6) (2018) 1555-1573. [4] P. Gill, J. Whitaker, C. Flaxman, N. Brown, J. Buckleton, An investigation of the rigor of interpretation rules for STRs derived from less than $100 \mathrm{pg}$ of DNA, Forensic science international 112(1) (2000) 17-40.

[5] P. Gill, H. Haned, O. Bleka, O. Hansson, G. Dørum, T. Egeland, Genotyping and interpretation of STR-DNA: Low-template, mixtures and database matches;Twenty years of research and development, Forensic Science International: Genetics 18 (2015) 100-117. 
[6] I.W. Evett, The interpretation of refractive index measurements, Forensic Science 9 (1977) 209217.

[7] M.G. Ensenberger, K.A. Lenz, L.K. Matthies, G.M. Hadinoto, J.E. Schienman, A.J. Przech, M.W. Morganti, D.T. Renstrom, V.M. Baker, K.M. Gawrys, M. Hoogendoorn, C.R. Steffen, P. Martin, A. Alonso, H.R. Olson, C.J. Sprecher, D.R. Storts, Developmental validation of the PowerPlex((R)) Fusion 6C System, Forensic Sci Int Genet 21 (2016) 134-44.

[8] O. Bleka, G. Storvik, P. Gill, EuroForMix: An open source software based on a continuous model to evaluate STR DNA profiles from a mixture of contributors with artefacts, Forensic Sci Int Genet 21 (2016) 35-44.

[9] D.J. Balding, J. Buckleton, Interpreting low template DNA profiles, Forensic Sci Int Genet 4(1) (2009) 1-10.

[10] P. Gill, H. Haned, A new methodological framework to interpret complex DNA profiles using likelihood ratios, Forensic Sci Int Genet 7(2) (2013) 251-63.

[11] D. Taylor, J.A. Bright, J. Buckleton, The interpretation of single source and mixed DNA profiles, Forensic Sci Int Genet 7(5) (2013) 516-28.

[12] M.W. Perlin, M.M. Legler, C.E. Spencer, J.L. Smith, W.P. Allan, J.L. Belrose, B.W. Duceman, Validating TrueAllele(R) DNA mixture interpretation, Journal of forensic sciences 56(6) (2011) 143047.

[13] R.G. Cowell, T. Graversen, S.L. Lauritzen, J. Mortera, Analysis of forensic DNA mixtures with artefacts, Journal of the Royal Statistical Society: Series C (Applied Statistics) 64(1) (2015) 1-48. [14] B. Haldemann, S. Dornseifer, T. Heylen, C. Aelbrecht, O. Bleka, H.J. Larsen, S. Willuweit, A. Alonso, J.M. Teodoridis, J. Morzfeld, L. Zatkalikova, M. Krupsky, B. Berger, W. Parson, N. Morling, P. Gill, U. Neuhaus-Steinmetz, eDNA;An expert software system for comparison and evaluation of DNA profiles in forensic casework, Forensic Science International: Genetics Supplement Series 5 (2015) e400-e402.

[15] H. Haned, Forensim: an open-source initiative for the evaluation of statistical methods in forensic genetics, Forensic Sci Int Genet 5(4) (2011) 265-8.

[16] H. Haned, P. Gill, Analysis of complex DNA mixtures using the Forensim package, Forensic Science International: Genetics Supplement Series 3(1) (2011) e79-e80.

[17] H. Haned, K. Slooten, P. Gill, Exploratory data analysis for the interpretation of low template DNA mixtures, Forensic Sci Int Genet 6(6) (2012) 762-74.

[18] C.C.G. Benschop, L. van de Merwe, J. de Jong, V. Vanvooren, M. Kempenaers, C.P. Kees van der Beek, F. Barni, E.L. Reyes, L. Moulin, L. Pene, H. Haned, T. Sijen, Validation of SmartRank: A likelihood ratio software for searching national DNA databases with complex DNA profiles, Forensic Sci Int Genet 29 (2017) 145-153.

[19] C.C.G. Benschop, J. Jong de, L. Merwe van de, H. Haned, Adapting a likelihood ratio model to enable searching DNA databases with complex STR DNA profiles, 27th International Symposium on Human Identification, 2016.

[20] R.C. Team, R: A Language and Environment for Statistical Computing. http://www.R-project.org/, 2014).

[21] $\varnothing$. Bleka, M. Bouzga, A. Fonneløp, P. Gill, dnamatch2: An open source software to carry out large scale database searches of mixtures using qualitative and quantitative models, Forensic Science International: Genetics Supplement Series 6 (2017) e404-e406.

[22] T. Graversen, DNAmixtures: Statistical analysis of DNA mixtures with artefacts. http://dnamixtures.r-forge.r-project.org).

[23] O. Garcia, J. Alonso, J.A. Cano, R. Garcia, G.M. Luque, P. Martin, I.M. de Yuso, S. Maulini, D. Parra, I. Yurrebaso, Population genetic data and concordance study for the kits Identifiler, NGM, PowerPlex ESX 17 System and Investigator ESSplex in Spain, Forensic Sci Int Genet 6(2) (2012) e78-9. [24] H. Haned, L. Pene, J.R. Lobry, A.B. Dufour, D. Pontier, Estimating the number of contributors to forensic DNA mixtures: does maximum likelihood perform better than maximum allele count?, Journal of forensic sciences 56(1) (2011) 23-8. 
[25] Ø. Bleka, G. Dørum, H. Haned, P. Gill, Database extraction strategies for low-template evidence, 744 Forensic Science International: Genetics 9 (2014) 134-141.

745 [26] O. Bleka, C.C.G. Benschop, G. Storvik, P. Gill, A comparative study of qualitative and quantitative models used to interpret complex STR DNA profiles, Forensic Sci Int Genet 25 (2016) 85-96. [27] J.M. Curran, P. Gill, M.R. Bill, Interpretation of repeat measurement DNA evidence allowing for multiple contributors and population substructure, Forensic science international 148(1) (2005) 4753.

750 [28] P. Gill, O. Bleka, T. Egeland, Does an English appeal court ruling increase the risks of miscarriages of justice when complex DNA profiles are searched against the national DNA database?, Forensic Sci Int Genet 13 (2014) 167-75.

[29] P. Gill, Misleading DNA evidence: Reasons for miscarriages of justice, Elsevier, London, 2014.

[30] Making Sense of Forensic Genetics, published by Sense about Science:

https://senseaboutscience.org/wp-content/uploads/2017/01/making-sense-of-forensic-genetics.pdf, (2017).

[31] P. Gill, T. Hicks, J.M. Butler, E. Connolly, L. Gusmao, B. Kokshoorn, N. Morling, R.A.H. van Oorschot, W. Parson, M. Prinz, P.M. Schneider, T. Sijen, D. Taylor, DNA commission of the International society for forensic genetics: Assessing the value of forensic biological evidence Guidelines highlighting the importance of propositions: Part I: evaluation of DNA profiling comparisons given (sub-) source propositions, Forensic Sci Int Genet 36 (2018) 189-202. [32] European Network of Forensic Science Institutes (ENFSI) guideline for evaluative reporting in forensic science. http://enfsi.eu/wp-content/uploads/2016/09/m1 guideline.pdf, 2015. [33] D. Taylor, J. Buckleton, Do low template DNA profiles have useful quantitative data?, Forensic Science International: Genetics 16 (2015) 13-16.

[34] R.S. Just, J.A. Irwin, Use of the LUS in sequence allele designations to facilitate probabilistic genotyping of NGS-based STR typing results, Forensic Sci Int Genet 34 (2018) 197-205.

[35] H. Haned, C.C.G. Benschop, P.D. Gill, T. Sijen, Complex DNA mixture analysis in a forensic context: evaluating the probative value using a likelihood ratio model, Forensic Sci Int Genet 16 (2015) 17-25.

[36] E. Alladio, M. Omedei, S. Cisana, G. D'Amico, D. Caneparo, M. Vincenti, P. Garofano, DNA mixtures interpretation - A proof-of-concept multi-software comparison highlighting different probabilistic methods' performances on challenging samples, Forensic Sci Int Genet 37 (2018) 143150.

[37] K. Slooten, Identifying common donors in DNA mixtures, with applications to database searches, Forensic Sci Int Genet 26 (2017) 40-47. 